\title{
Swarm Intelligence based Optimization in Thermal Image Fusion using Dual Tree Discrete Wavelet Transform
}

\author{
by K.Madheswari ${ }^{1}$ and N.Venkateswaran ${ }^{2}$
}

\author{
${ }^{1}$ Department of Computer Science and Engineering, SSN College of Engineering, Chennai, India, \\ madheswarik@ssn.edu.in \\ ${ }^{2}$ Department of Electronics and Communication Engineering,SSN College of Engineering,Chennai,India, \\ venkateswarann@ssn.edu.in
}

\begin{abstract}
In this paper, we propose a Particle Swarm Optimized image fusion framework in Discrete Wavelet Transform domain that combines the thermal image with the visual image to obtain a single informative fused image. Dual tree Discrete Wavelet Transform (DT-DWT) is applied for feature selection and particle swarm optimization (PSO) technique is used to obtain the optimized image. In the fusion process, an optimized weighting factor has been used to form a new composite image with maximized Entropy and minimized Root Mean Square Error (RMSE). Simulation results indicate that the proposed fusion framework results in better quality of the fused image.
\end{abstract}

Keywords: Image fusion, Dual Tree Discrete Wavelet Transform, Particle Swarm Optimization, Entropy, Root Mean Square Error.

\section{Introduction}

Recently, Infrared imaging (IR) systems have gained importance in many application areas such as medicine, industry , military etc., Thermal infrared image is formed due to emission of heat against visual band images that are formed due to light reflection $[1,2,3]$. Visual band imaging is highly matured and successful due to the advancement of sensor technology, availability of computing power, and high digital storage capacity $[4,5,6]$. However, the image processing task and applications are challenging if the visual images obtained under non-ideal environments $[7,8,9]$. The end result depends on the extent of external light source, which sometimes might be absent in environments when there are heavy clouds, fog, rain, snow, smoke, darkness, shadows and at night-time.

In contrast, Thermal imaging does not depend on any external light source. It is robust against any illumination changes. But, IR imaging is sensitive to temperature changes in the surrounding environment. Currents of cold or warm air could influence the performance of the IR system $[4,5,9]$. IR imaging is also sensitive to variations in the heat patterns of the objects. Thus, IR and visual imaging posses highly complementary strengths and limitations. It is therefore, advantageous to acquire and process both visual and IR images concurrently and jointly in many applications leading to the fusion of information.

Image fusion is the process of blending the most pertinent information from multiple source images to obtain an accurate fused image. The fused image is formed to get a better image content and make it easier for further image processing tasks. The fused image contains much richer and more accurate information content, which is advantageous to the analysis and processing of image signal. It makes the human observation easier and more suitable for computers in image processing applications [10]. The main disadvantages of contemporary image fusion techniques is that they simply combine the image data together without weighing or analysing the source images and result in information loss affecting the spectral characteristics of an image. This kind of techniques may not be acceptable in many cases. In order to advance the previously used image fusion methods, in this paper we have introduced an optimized image fusion framework which will produce an accurately fused image with the aim of displaying the most significant features from the visible and thermal images. The objectives of this study were to combine visible and thermal images using optimized image fusion rule to maximize the information content and minimize the root mean square error.

The rest of the section is organized as follows. Section 2 describes the technical background and proposed framework. Results and discussions are given in section 4, finally conclusions are drawn at section 5.

\section{Technical Background}

\subsection{Image Fusion Techniques}

One of the common approaches towards image fusion is the pixel-level image fusion and fusion takes place specifically at the pixel intensities. It is advantageous over the other fusion schemes such as feature level and decision 
level, as it uses original (pixel values) information of images and can be performed both in spatial or transform domain. Thus, spatial domain fusion directly operates on the pixels of the source images. Some of the technique falls under this category is discussed below.

\subsubsection{Simple Averaging}

Simple averaging is a very basic and straightforward technique and image fusion is achieved by computing the average intensity of corresponding pixels from both the input images [11].

$$
F(x, y)=\frac{A(x, y)+B(x, y)}{2}
$$

Where, $A(x, y), B(x, y)$ are input images and $F(x, y)$ is the fused image. However when this method is applied, the contrast features exclusively presented in either of the images is tremendously decreased. Because of the averaging operation, both the good and bad information are minimized in the fused image.

\subsubsection{Simple minimum}

In this approach, image fusion could be achieved by choosing the minimum intensity of corresponding pixels from both the input images [11].

$$
F(x, y)=\sum_{x=0}^{m} \sum_{y=0}^{n} \min (A(x, y), B(x, y))
$$

This methodology is suitable for the images with dark shades and would produce a high-quality fused image.

\subsubsection{Simple maximum}

In this technique, image fusion could be achieved by choosing the maximum intensity of corresponding pixels from both the input images.

$$
F(x, y)=\sum_{x=0}^{m} \sum_{y=0}^{n} \max (A(x, y), B(x, y))
$$

The simple maximum approach gives a major benefit over averaging method, and there is no negotiation made over the good information present in the input images. Although a direct selection of pixel with higher intensity is made here, chose of higher pixel intensity does not necessarily mean better information [11].

\subsubsection{Principal Component Analysis (PCA)}

By applying PCA on input images that translates a number of correlated variables into uncorrelated variables. The input images are arranged in two column vectors and their empirical means are subtracted. The resulting vector has a dimension of $n \times 2$, where $\mathrm{n}$ is length of each image vector. Eigen vector $(V)$ and Eigen value $(D)$ for the resulting vector is computed and obtain the Eigen vectors corresponding to the larger Eigen values.

The fused image is obtained by

$$
F(x, y)=P_{1} A(x, y)+P_{2} B(x, y)
$$

Where, $P_{1}$ and $P_{2}$ are normalized principal components, computed as follows

$$
P_{1}=\frac{D(1)}{\sum V} \quad \text { and } \quad P_{2}=\frac{D(2)}{\sum V}
$$

Where, $D(1)$ and $D(2)$ are the first and second larger Eigen values in the Eigen vector $(V)$.By using this image fusion technique redundancy of the image data can be decreased, but it produces spectral degradation in the fused image $[8,11]$.

\subsubsection{Pyramid based fusion methods}

Pyramid and wavelet transforms are the most popular multi-resolution methods for pixel level fusion. In pyramid transform based image fusion scheme, source images are decomposed into their pyramidal representations followed by application of fusion rules to pyramidal representation. Inverse pyramid transform will give resultant fused image. Gaussian pyramid [12], Laplacian pyramid [13], Gradient pyramid [14], Contrast pyramid [15], Ratio of low pass pyramid [16], Filter subtract decimate pyramid [17], Morphological pyramid [17] are used for image fusion. Pyramid transform based fusion methods suffer from blocking effect [18] in the regions where the input images are significantly different. Also pyramid transform based fusion methods do not provide any directional information and have poor Signal to Noise Ratio (SNR). 


\subsubsection{Wavelet based fusion methods}

Discrete wavelet transform (DWT) is the most regularly used wavelet transform for image fusion. It provides spectral as well as improved directional information with three spatial orientations namely vertical, horizontal and diagonal. The most extensively used form of the DWT is the DDWT implemented in a multi-resolution decomposition/reconstruction filter bank tree. The non-redundant nature and ideal reconstruction properties of the DDWT are mainly eye-catching for applications such as signal/image compression and noise reduction. The DDWT has two drawbacks: Sensitivity to input signal shifts and constrained directionality $[19,20]$. A time-shift in the input signal can result in variations in the DDWT coefficients. This sensitivity is because of the down sampling process in decomposition and results in aliased artifacts in the down sampled signals and images. As a consequence of shift sensitivity the DDWT coefficients cannot distinguish between input signal shifts [21,22].

The UDWT has the similar filter bank structure as the DDWT except the down sampling process is disposed of. The UDWT does not encompass the shift sensitivity of the DDWT. In whatever way, the approximation and detail signals at each one level of the UDWT are the identical size as the original signal resulting in very high redundancy. The DTDWT is a late variety of the DDWT $[23,24,25]$. The DT DWT was actualized to conquer the shift variance and constrained directionality of the DDWT while keeping the ideal reconstruction property with restricted redundancy.

\subsection{Applications}

Image fusion is the important component in the development of intelligent machines and systems. The successful application of image fusion will lead to improved performance of military surveillance [2], remote sensing, medical imaging [14], face recognition [3,5,7,10,11], situational awareness, agriculture, concealed weapon detection[20] and satellite imaging.

\section{PSO Optimized Image Fusion Framework}

In this section we present the proposed image fusion algorithm. Visible image and thermal image was captured using different cameras hence the source images have different fields of view and spatial resolutions. Image Preprocessing is performed on both source images (i.e. image resampling) since a pixel-level approach was used, the source images were registered using affine transform. The visible image was selected as the base image and the thermal image was the input image; i.e. the image to be registered.

After image registration the source images are decomposed into wavelet coefficients using Dual-Tree Discrete Wavelet Transform (DT-DWT). In the fusion process an optimized weighting factor has been used to form a new composite image with maximized Entropy and minimized Root Mean Square Error (RMSE). We proposed an optimized weighted averaging rule in the fusion of the wavelet coefficients. Swarm intelligence based Particle Swarm Optimization (PSO) is used to find the optimal weights with Entropy and RMSE based objective function. The wavelet coefficients of the source images are fused based on the optimal weights obtained and on application of inverse DT-DWT the resultant fused image is obtained. Figure 1 demonstrates the block diagram of the proposed method, in which particle swarm optimization framework is employed in calculating the optimal weights in order to best enhance the fused image. The proposed algorithm is given in figure 2 .

\subsection{Dual Tree Discrete Wavelet Transform (DT-DWT)}

The dual tree discrete wavelet transform (DT-DWT) was introduced to surmount shift invariance and directionality restrictions of the DWT, while sustaining the perfect reconstruction property with limited redundancy $[25,26$, 27,28]. The DT-DWT is made out of two parallel DWT filter bank trees; the first tree gives the real part of the transform whereas the second tree gives the imaginary part. The wavelet and scaling functions utilized in one tree can be described as approximate Hilbert transforms of the functions in the other tree. The filters utilized in both trees are real, but the united filters are said to be analytic. The successful process of the DT-DWT is focused around the distinction between the filters in the two trees. In this paper, the DT-DWT of an image $d$ is denoted by $f$ and is assumed in the different scales to be of the form: $f=\left\{f^{1}, f^{2}, f^{3}, \ldots, f^{\ell}, d^{\ell}\right\}$. Here, $\mathrm{d}^{\ell}$ represents the approximation or low frequency subbands at the last decomposition level, while $f^{\ell}$ represents the details or high frequency sub-bands at level $\ell$. In addition, $f^{\ell}$ is composed of twelve directional sub-bands, six of which are real, and six are imaginary as given as follows.

$$
f^{\ell}=\left\{\begin{array}{l}
\text { realfreal }^{\ell}\left(i, \frac{j}{1}\right), \text { freal }^{\ell}\left(i, \frac{j}{2}\right), \ldots, \text { freal }^{\ell}\left(i, \frac{j}{6}\right) \\
\text { imaginaryfimg }^{\ell}\left(i, \frac{j}{1}\right), \text { fimg }^{\ell}\left(i, \frac{j}{2}\right), \ldots, \text { fimg }^{\ell}\left(i, \frac{j}{6}\right)
\end{array}\right\}
$$


The sources images A and B are decomposed into approximation (low frequency) and detailed (high frequency) components at the required level using DT-DWT. The coefficients of the visual and thermal images are subsequently combined using the fusion rule. The fused image $f$ is then obtained by taking the inverse DT-DWT to the fused coefficients.

$$
F=T^{-1}[\varphi\{T(A), T(B)\}]
$$

Where $T$ is the DT-DWT. The fusion rule $(\varphi)$ used here is,

$$
\varphi=w_{1} \times A(x, y)+w_{2} \times B(x, y)
$$

Here $w_{1}$ and $w_{2}$ determine the percentage of each image displayed in the fused image, without any information loss and change in spectral characteristics of an image. Many of the fusion rules are based on fixed weights which do not suitable for all kind of images. Therefore it is necessary to find an optimal weights for fusion of wavelet coefficients that lead to maximized information and minimized root mean square error.

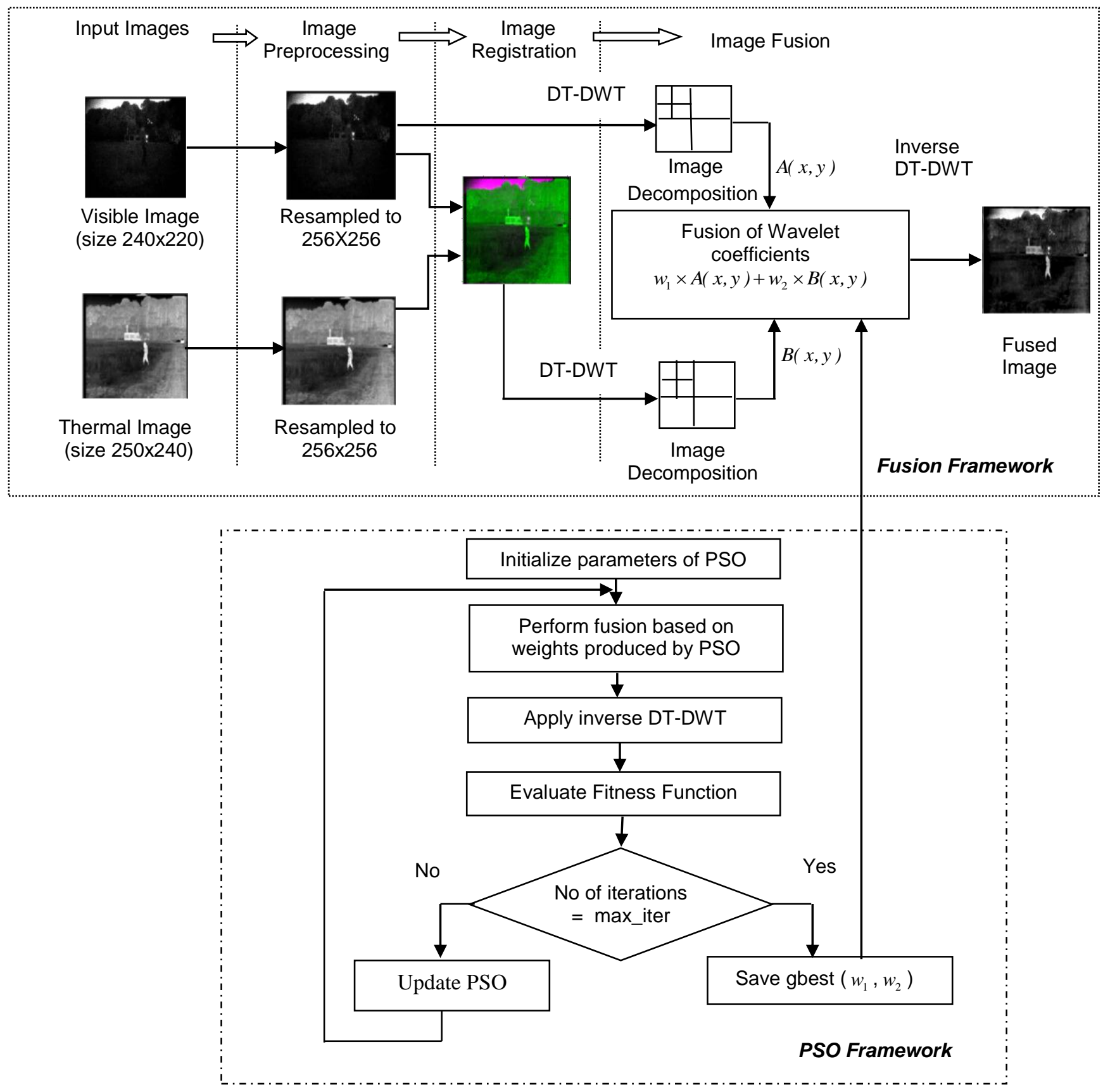

Fig. 1. PSO optimized image fusion framework 


\section{Algorithm for proposed image fusion approach}

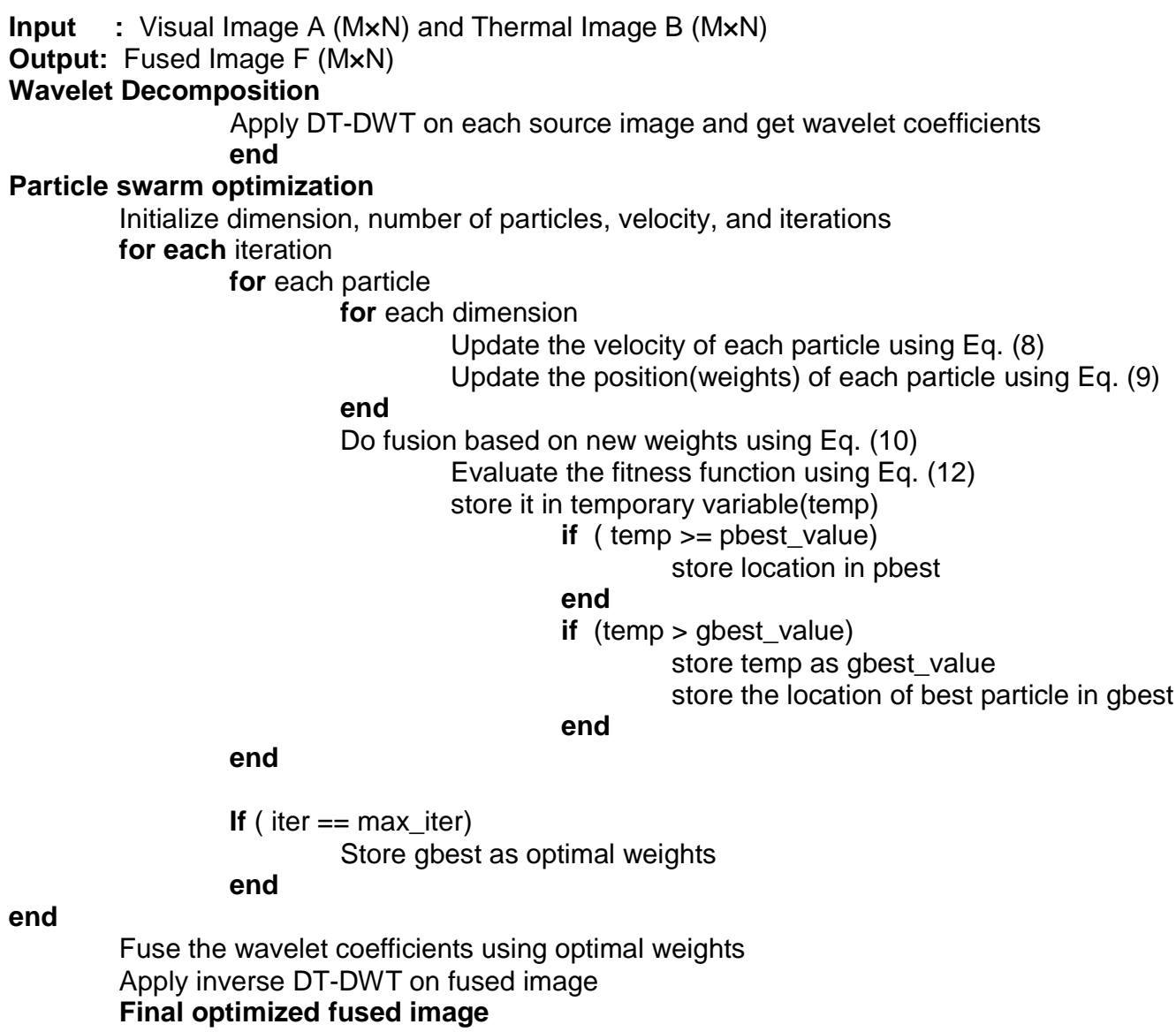

end

Fuse the wavelet coefficients using optimal weights

Apply inverse DT-DWT on fused image

Final optimized fused image

Fig. 2. PSO optimized image fusion Algorithm.

\subsection{Particle Swarm optimization (PSO)}

PSO is a population -based optimization technique with the aim of exploiting the population of possible solutions to probe the search space simultaneously. In a mathematical context PSO can be stated as follows. Let

$$
A \subset R^{n} \text { and } f: A \rightarrow Y \subseteq R
$$

Where, $A$ the search is space and $f$ be the objective function. The whole population is called as swarm and its individuals are called particles. The swarm is defined as a set:

of $N$ particles(possible solutions), defined as:

$$
S=\left\{x_{1}, x_{2}, \ldots, x_{N}\right\},
$$

$$
x_{i}=\left(x_{i 1}, x_{i 2}, \ldots, x_{i d}\right)^{T} \in A \quad i=1,2, \ldots, N
$$

Indices are randomly assigned to particles, where $N$ is a user defined parameter of the algorithm and $\mathrm{d}$ be the dimension. The Each particle has a unique fitness function value:

$$
f_{i}=f\left(x_{i}\right) \in Y
$$

The particles are assumed to move inside the search space iteratively by adjusting their position using a proper location shift called velocity, indicated as:

$$
v_{i}=\left(v_{i 1}, v_{i 2}, \ldots, v_{i d}\right)^{T}, \quad i=1,2, \ldots, N
$$

Velocity is updated iteratively to make the particles efficiently move in any region of $A$. If $t$ represents the iteration count, then the current position of the $i^{\text {th }}$ particle and its velocity is indicated as $x_{i}(t)$ and $v_{i}(t)$ respectively. Velocity of the particle is updated based on the information gained from previous steps of the algorithm [29]. PSO also has a memory set:

$$
P=\left\{P_{1}, P_{2}, \ldots, P_{N}\right\}
$$


This contains the best positions:

$$
P_{i}=\left(P_{i 1}, P_{i 2}, \ldots, P_{i d}\right)^{T} \in A, \quad i=1,2, \ldots, N
$$

Ever visited by each particle. These positions are defined as

$$
P_{i}(t)=\arg _{t} \max f_{i}(t),
$$

PSO is modelled based on the social behaviour of birds that allows particles to mutually communicate their experience. As a result the algorithm calculates the global best position which is ever visited by swarm. The global best position is denoted by index $\mathrm{g}$. i.e. (highest fitness function value in $\mathrm{P}$ at a given iteration $t$ ),

$$
P_{g}(t)=\arg _{t} \max f\left(P_{i}(t)\right)
$$

The velocity and positions of each particle updated during each iteration by applying the following equation

$$
\begin{gathered}
v_{i}(t+1)=\omega v_{i}(t)+c_{1} r_{1}\left(P_{i}(t)-x_{i}(t)\right)+c_{2} r_{2}\left(P_{g}(t)-x_{i}(t)\right) \\
x_{i}(t+1)=x_{i}(t)+V_{i}(t+1)
\end{gathered}
$$

Where, $\omega$ is called as the inertia weight that control the convergence behaviour of PSO. The large inertia weight aids global search (searching new areas), while small inertia weight tends to aid local search. The parameters $c_{1}$ and $c_{2}$ are stabilizes the influence of the individual best and global best position. The parameters $r_{1}$ and $r_{2}$ are applied to control the diversity of the population, and they are distributed in the range [0, 1].

\subsection{Optimization of Image Fusion Rule}

In our proposed work the image is decomposed using DT-DWT, the decomposed wavelet coefficients should be fused based on fusion rule. The fusion rule is given as

$$
F(x, y)=w_{1} \times A(x, y)+w_{2} \times B(x, y)
$$

Where, $A(x, y)$ and $B(x, y)$ are input wavelet coefficients and $F(x, y)$ is fused wavelet coefficients. PSO is employed to find optimal weights $w_{1}$ and $w_{2}$ which maximizes the entropy and minimizes the root mean square error. An image with high entropy (high information content) and RMSE improves the fusion performance. In this paper image fusion is formulated as an optimization problem as given below: The set of particle values (weights) is defined as a set of $N$ particles

Where,

$$
w=\left\{\begin{array}{l}
w_{11}, w_{12}, \ldots, w_{1 N} \\
w_{21}, w_{22}, \ldots, w_{2 N}
\end{array}\right\}
$$

$$
w=\left(w_{1}, w_{2}\right)^{T} \in A
$$

Which maximizes the objective function (Entropy)

$$
\arg _{t} \max (H)=-\sum_{i=0}^{255} p(i) \times \log _{2}(p(i))
$$

Where $p(i)$ is the probability of occurrence of $i^{\text {th }}$ intensity of the fused image. The particle set $w$ should also minimize the objective function (Root Mean square Error)

$$
\arg _{t} \min (R M S E)=\frac{1}{2}\left[\sqrt{\frac{1}{M N} \sum_{i=1}^{M} \sum_{j=1}^{N}[F(i, j)-V(i, j)]^{2}}+\sqrt{\frac{1}{M N} \sum_{i=1}^{M} \sum_{j=1}^{N}[F(i, j)-T(i, j)]^{2}}\right]
$$

$F(i, j), V(i, j)$ and $T(i, j)$ are fused, visible and thermal images respectively. A multi-objective optimization technique usually applies a method called pareto dominance [29], but this may increase the computational complexity. One of the simple ways to handle the multi-objective optimization problem is to construct an overall objective function as a linear combination of the multiple conflicting objective functions [29]. The overall objective function is defined as follows: 


$$
f(x)=\alpha_{1} f_{1}(x)+\alpha_{2} f_{2}(x)
$$

Here, $\alpha_{1}$ and $\alpha_{2}$ are constants whose values indicates the relative significance of one objective function relative to other. In our proposed work two contrasting objectives are included; (i) Minimization of RMSE (ii) The maximization of information content in the image. This observation matches with the structure of the standard PSO and is applied to incorporate the optimization of a multiple goals leading to the multi-objective particle swarm optimization. The proposed multi objective function is formulated as follows:

$$
\left.f(x)=\alpha_{1}\left[-\sum_{i=0}^{255} p(i) \times \log _{2}(p(i))\right]+\alpha_{2}\left[\frac{1}{\frac{1}{2}\left[\sqrt{\frac{1}{M N} \sum_{i=1}^{M} \sum_{j=1}^{N}[F(i, j)-V(i, j)]^{2}}+\sqrt{\frac{1}{M N} \sum_{i=1}^{M} \sum_{j=1}^{N}[F(i, j)-T(i, j)]^{2}}\right.}\right]\right]
$$

Each row in the particle set is substituted in Eq. (10) and the fused image is obtained. The solution set which gives the maximum entropy value and minimum root mean square error for the fused image will be stored as gbest. After the maximum number of iterations is reached the gbest value is used to get the final fused image.

\subsection{Evaluation of Image Fusion}

Metrics are considered in this paper, which do not require ground truth (reference images) for evaluation. Standard deviation is the square root of the variance, which reflects the spread in information and is given by

$$
\sigma=\sqrt{\sum_{0}^{255}\left(i-i_{\text {mean }}\right)^{2} h(i)}
$$

Where, $i$ and $i_{\text {mean }}$ is gray-level and mean intensity of the image, and $h(i)$ is the normalized histogram of the image. It is well-known that standard deviation is made out of the signal and noise parts. This metric would be more productive without noise. It gauges the contrast in the fused image hence; an image with high contrast would have a high standard deviation.

Spatial Frequency is used to measure the action level in an image. A large value of spatial frequency depicts the vast activity level in the image which represents the clarity of the image.

$$
\begin{gathered}
S F=\sqrt{(R F)^{2}+(C F)^{2}} \\
R F=\sqrt{\frac{1}{m \times n} \sum_{i=1}^{m} \sum_{j=2}^{n}[F(i, j)-F(i, j-1)]^{2}} \\
C F=\sqrt{\frac{1}{m \times n} \sum_{i=1}^{m} \sum_{j=2}^{n}[F(i, j)-F(i-1, j)]^{2}}
\end{gathered}
$$

Here $R F$ and $C F$ are row frequency and column frequency. $F$ is the fused image; $m \times n$ is the size of the fused image.

\section{Results and Discussion}

The proposed image fusion framework was tested against several state-of-the-art image fusion methods namely simple averaging, simple minimum, PCA, and DWT. The proposed fusion method is implemented using MatLab 2013. Figure 3,4,5 shows the result of different image fusion algorithms for the forest, room and street image respectively. Subjective or visual comparison between different methods indicates superiority of the proposed method against other image fusion algorithms. The fused image is similar to visible image while applying the minimum selection method on the forest image. The reason behind the result is that, the visual image is having the dark background which contains low intensity value. Maximum selection approach produces the result which is replica of thermal image and averaging approach gives best result compared with the above two methods. Despite of the result obtained from averaging method, it is observed that the intensity level of the human and building in the forest image is low. In PCA method, the source image transposed completely in resulting image and information retrieved from that fused image not good as DWT. The DWT fused image has low contrast between the forest and human in compared with proposed method. The result obtained for the street and room images by proposed method gives more information than the existing fusion methods. The optimal weights and fitness value for each image is given in table 1 . The PSO parameters selected for the optimization problem are listed in table 2 . 


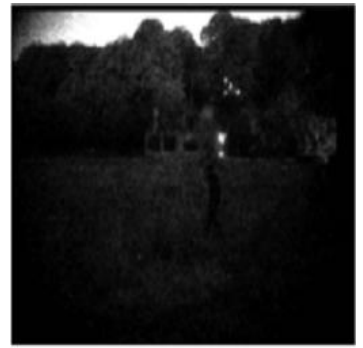

(a) Visible Image

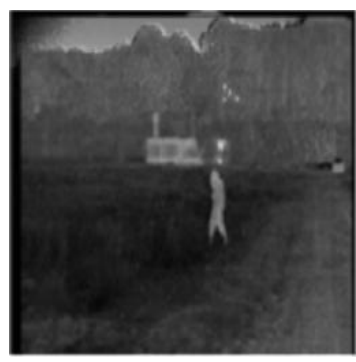

(e) Averaging

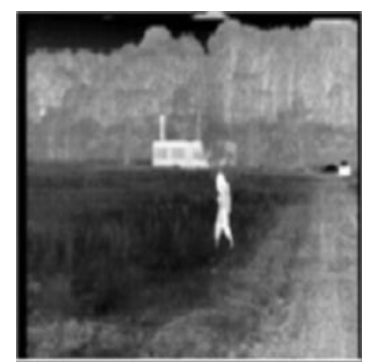

(b) Thermal Image

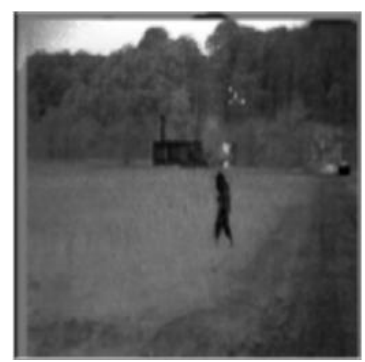

(f) PCA

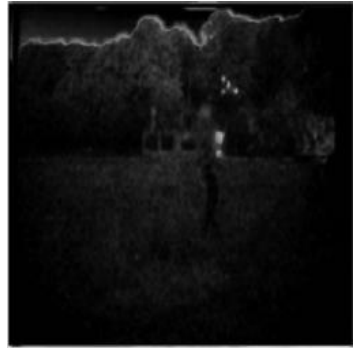

(c) Minimum selection

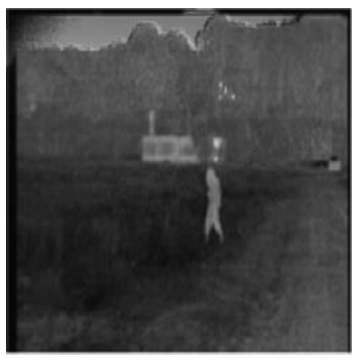

(c) DWT

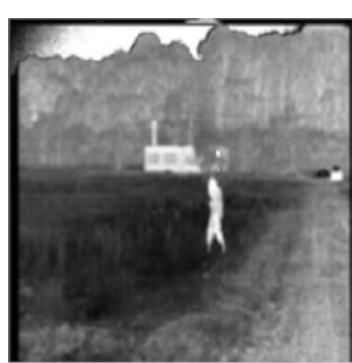

(d) Maximum Selection

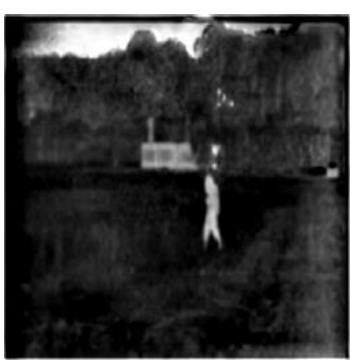

(d) Proposed

Fig. 3. Fused image of forest image using various image fusion algorithm.

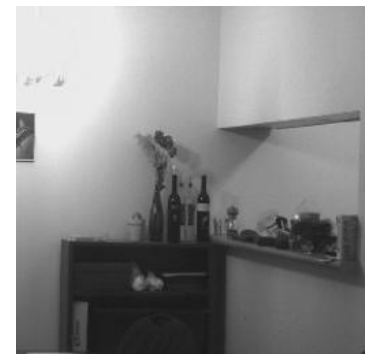

a) Visible Image

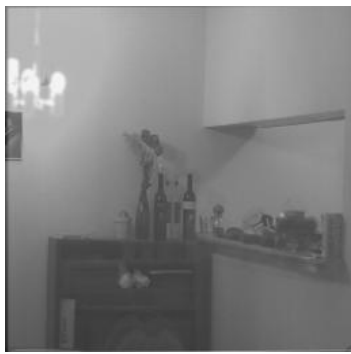

(e) Averaging

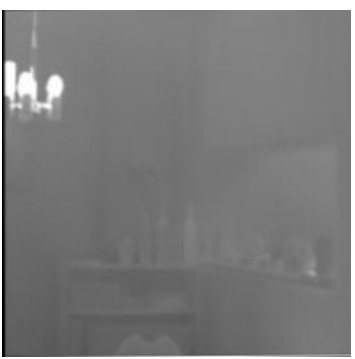

(b) Thermal Image

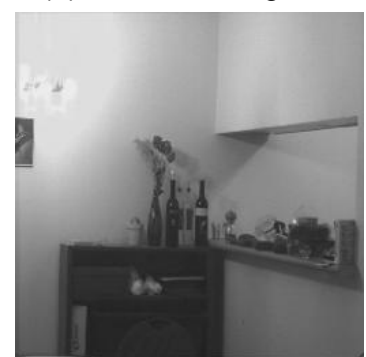

(f) PCA

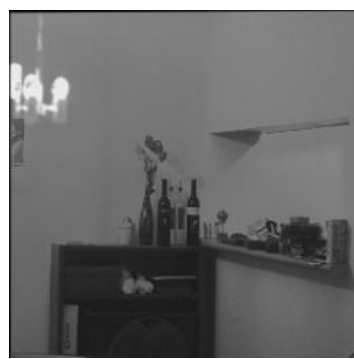

(c) Minimum selection

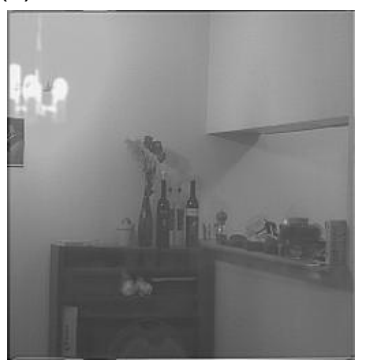

(c) DWT

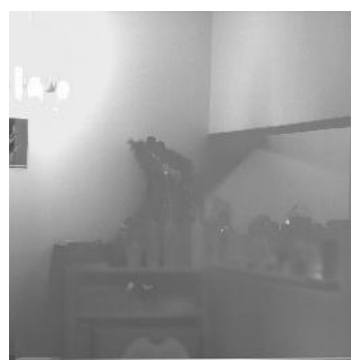

(d) Maximum Selection

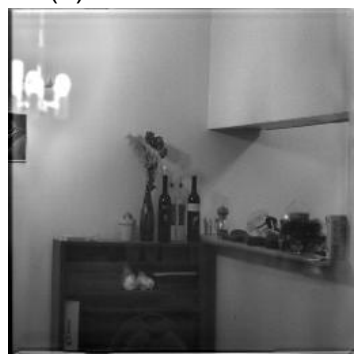

(d) Proposed

Fig. 4. Fused image of room image using various image fusion algorithm

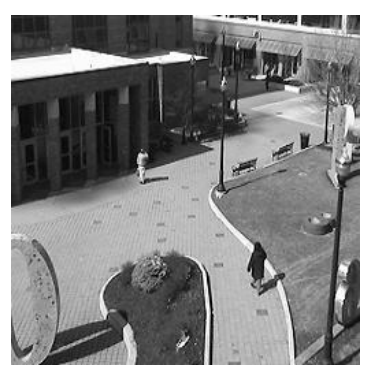

a) Visible Image

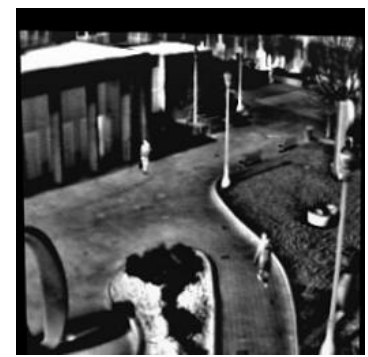

(b) Thermal Image

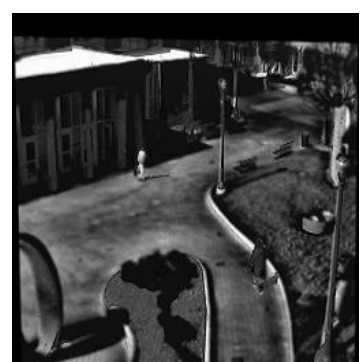

(c) Minimum selection

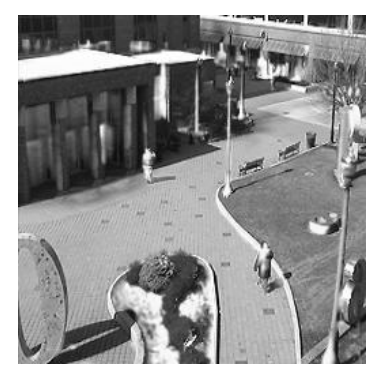

(d) Maximum Selection 


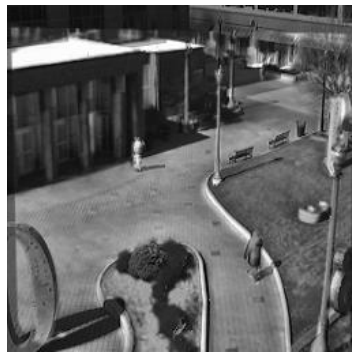

e) Averaging

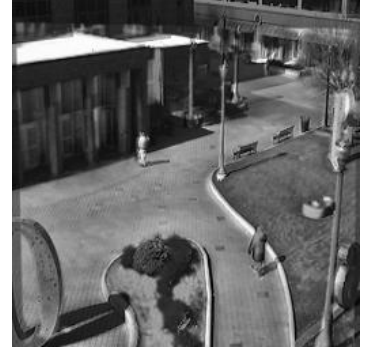

(f) PCA

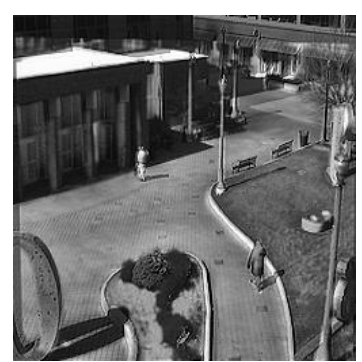

(c) DWT

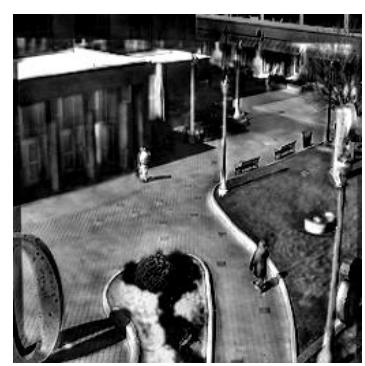

(d) Proposed

Fig. 5. Fused image of street image using various image fusion algorithm

Table 1. Optimal weights

\begin{tabular}{|l|l|l|l|}
\hline Image & W1 & W2 & $\begin{array}{l}\text { Fitness } \\
\text { value }\end{array}$ \\
\hline Forest & 0.62 & 0.38 & 1.521 \\
\hline Room & 0.92 & 0.08 & 1.114 \\
\hline Street & 0.85 & 0.15 & 1.523 \\
\hline
\end{tabular}

Table 2. Parameters of PSO

\begin{tabular}{|l|l|}
\hline PSO parameters & Values \\
\hline $\begin{array}{l}\text { Particle size(N), } \\
\text { Dimension }(\mathrm{D}) \text { and Iteration }\end{array}$ & 50,2 and 80 \\
\hline Inertia $(\omega)$ & $\begin{array}{l}\omega \text {-initial }=0.9 \\
\omega \text {-final }=0.4\end{array}$ \\
\hline Learning Factors $\left(\mathrm{C}_{1}, \mathrm{C}_{2}\right)$ & $\mathrm{C}_{1}=2, \mathrm{c}_{2}=2$ \\
\hline
\end{tabular}

The metric qualities demonstrate the subjective assessment, that our proposed image fusion algorithm generally incorporates more data from the source images into the fused images. Table 3 reflects the performance of fusion. The entropy index and root mean square error for the proposed method is better compared to other fusion method, which indicates that the fused image obtained by the proposed method has more information content compared to the fused images using other fusion algorithms.

Table 3. Quantitative Analysis of image quality metrics

\begin{tabular}{|c|c|c|c|c|c|c|}
\hline Measure/Technique & $\begin{array}{l}\text { Proposed } \\
\text { Approach }\end{array}$ & DWT & PCA & Averaging & minimum & maximum \\
\hline \multicolumn{7}{|c|}{ Result of Forest Image } \\
\hline Entropy(E) & 7.55 & 6.86 & 0.76 & 6.85 & 5.43 & 7.55 \\
\hline RMSE & 6.62 & 9.42 & 4.34 & 9.38 & 2.34 & 9.71 \\
\hline $\begin{array}{c}\text { Standard } \\
\text { Deviation(SD) }\end{array}$ & 56.20 & 35.83 & 522.64 & 34.96 & 27.79 & 57.15 \\
\hline Spatial frequency(SF) & 24.20 & 17.67 & 160.03 & 14.02 & 19.01 & 17.40 \\
\hline \multicolumn{7}{|c|}{ Result of Room image } \\
\hline Entropy(E) & 7.56 & 7.07 & 7.38 & 7.02 & 6.38 & 6.87 \\
\hline RMSE & 6.78 & 9.97 & 7.87 & 9.96 & 1.04 & 10.69 \\
\hline $\begin{array}{c}\text { Standard } \\
\text { Deviation(SD) }\end{array}$ & 56.61 & 38.31 & 57.92 & 37.56 & 37.69 & 44.44 \\
\hline Spatial frequency (SF) & 14.76 & 11.59 & 7.95 & 6.57 & 10.46 & 7.11 \\
\hline \multicolumn{7}{|c|}{ Result of Street image } \\
\hline Entropy(E) & 7.67 & 7.51 & 7.42 & 7.43 & 6.76 & 7.63 \\
\hline RMSE & 8.85 & 9.70 & 9.80 & 9.82 & 2.05 & 10.23 \\
\hline $\begin{array}{c}\text { Standard } \\
\text { Deviation(SD) }\end{array}$ & 70.30 & 53.90 & 51.44 & 51.32 & 50.08 & 62.95 \\
\hline Spatial frequency(SF) & 2.05 & 35.93 & 24.29 & 23.87 & 24.92 & 35.33 \\
\hline
\end{tabular}

\section{Conclusion}

This paper presented an optimized image fusion framework to combine thermal and visible image to produce a single informative fused image. Prior to image fusion thermal image is registered by taking visual image as a reference image. The proposed method applied the DT-DWT for image decomposition and Swarm intelligence based Particle Swarm Optimization (PSO) is used to find the optimal weights with Entropy and RMSE based objective function. The wavelet coefficients of the source images are fused based on the optimal weights obtained and on application of inverse DT-DWT the resultant fused image is obtained. The experimental results demonstrates that the proposed methodology has more entropy index which shows that it integrates more information content from the source images into fused image. Comparing with other methods, our fusion method is superior in terms of both quantitatively and visually because it combines the most significant features from the source images. 


\section{REFERENCES}

[1] Stephen R.,Schnelle and Alex L.Chan., "Fusing Infrared and visible imageries for improved tracking of Moving targets", Army Research Labortary, ARL-TR-5552, July 2011.

[2] Amar El-Maadi., Vincent Grégoire., Louis St-Laurent., Hélène Torresan., Benoit Turgeon., Donald Prévost., Patrick Hébert., Denis Laurendeau., Benoit Ricard., and Xavier Maldague.,"Visible and infrared imagery for surveillance applications: software and hardware considerations", QIRT Journal, vol. 4, pp. 25-40, 2007.

[3] Prokoski F., Riedel R., "Infrared Identification of Faces and Body Part", In BIOMETRICS Personal Identification in networked Society, Kluwer Academic Publishers,1998.

[4] Stephen R. Schnelle, Alex Lipchen Chan, "Enhanced Target Tracking Through Infrared-Visible Image Fusion", 14th International Conference on Information Fusion Chicago, Illinois, USA, July 5-8, 2011

[5] George Bebis a., Aglika Gyaourova a, Saurabh Singh a, loannis Pavlidis b, "Face recognition by fusing thermal infrared and visible imagery", Image and Vision Computing, vol- 24 pp. 727-742, 2006.

[6] Jamal Saeedi., Karim Faez., "Infrared and visible image fusion using fuzzy logic and population-based optimization", Applied Soft Computing, vol-12, pp. 1041-1054 , 2012

[7] R. Raghavendra a, Bernadette Dorizzi a , Ashok Rao b , G. Hemantha Kumar c ," Particle swarm optimization based fusion of near infrared and visible images for improved face verification", Pattern Recognition, vol-44, pp. 401-411, 2011

[8] Rajiv Singh., Ashish Khare.,Rajiv Singh., Ashish Khare., "Fusion of multimodal medical images using Daubechies complex wavelet transform - A multiresolution approach", Information Fusion, vol-19, pp. 4960,2014

[9] Prokoski F., " History, current status, and future of infrared identification", Proceedings of IEEE Workshop on Computer Vision Beyond the Visible Spectrum: Methods and Applications, Hilton Head Island(South Carolina),pp. 5-14, 2000.

[10] George Bebis a., Aglika Gyaourova a., Saurabh Singh a., Ioannis Pavlidis., "Face recognition by fusing thermal infrared and visible imagery", Image and Vision Computing Journal, vol. 24, pp. 727-742, 2006.

[11] Tumpa Dey., "A Survey on Different Fusion Techniques of Visual and Thermal Images for Human Face Recognition", International Journal of Electronics Communication and Computer Engineering, Vol-4, 2013.

[12] V.P.S. Naidu., J.R. Raol., "Pixel-level image fusion using wavelets and principal component analysis", Defence Science Journal, vol. 58, pp. 338-352, 2008.

[13] Y. Chibani., "Additive integration of SAR features into multispectral SPOT images by means of the atrous wavelet decomposition" , ISPRS Journal of Photogrammetry \& Remote Sensing, vol. 60, pp. 306-314, 2006.

[14] S. Daneshvar., H. Ghassemian., "MRI and PET image fusion by combining IHS and retina-inspired models", Information Fusion, vol. 11, pp. 114-123, 2010.

[15] Olkkonen, H., and pesola, P., "Gaussian pyramid Wavelet Transform for Multi-resolution analysis of images", Graphical models and Image Processing, vol.58, pp.394-398,1996.

[16] P. Burt, E. Adelson, "The laplacian pyramid as a compact image code", IEEE Transactions on Communications vol-31, pp. 532-540, 1983.

[17] A. Toet, J.J. Van Ruyven, J.M. Valeton, "Merging thermal and visual images by a contrast pyramid", Optical Engineering, vol-28, pp. 789-792,1989.

[18] A. Toet, "Image fusion by a ratio of low-pass pyramid", Pattern Recognition Letters, vol-9, pp. 245-253,1989.

[19] H.Anderson, "A filter- subtract-decimate hierarchical pyramid signal analysing and synthesizing technique," U.S.Patent 718 104, 1987.

[20] Ramac, L.C., Uner, M.K, Varshney,P.K., "Morphological filters and wavelet based image fusion for concealed weapon detection," Proceedings of SPIE, Vol. 3376,1998.

[21] H. Li, B.S. Manjunath, S.K. Mitra, "Multi-sensor image fusion using the wavelet transform", Graphical Models and Image Processing, vol-57,pp. 235-245, 1995.

[22] S. Mallat, "A Wavelet Tour of Signal Processing", Academic Press, London, 1998.

[23] H. J. Heijmans and J. Goutsias, " Multiresolution signal decomposition schemes, Part 2: Morphological wavelets", IEEE Trans. Image Processing, vol.9, pp.1897-1913, 2000.

[24] A. Toet, J.J. Van Ruyven, J.M. Valeton, "Merging thermal and visual images by a contrast pyramid", Optical Engineering, vol-28, pp. 789-792. 1989.

[25] Kingsbury, N.G., "Image Processing with Complex Wavelets," Phil. Trans. Royal Society, 1999.

[26] Kingsbury, N.G., "A Dual-Tree Complex Wavelet Transform with Improved Orthogonality and Symmetry Properties," Proc. IEEE Conf. on Image Processing, 2000

[27] Kingsbury, N.G., "Complex Wavelets for Shift Invariant Analysis and Filtering of Signals," Journal of Applied and Computational Harmonic Analysis, 10(3),234-253, 2001.

[28] Kingsbury, N.G., "Design of Q-shift Complex Wavelets for Image Processing using Frequency Domain Energy Minimization," Proc. IEEE Conf. on Image Processing, 2003.

[29] Lili Liu., DingweiWang., shengxiang Yang.,"Compound Particle Swarm Optimization in Dynamic Environments" EvoWorkshops 2008, LNCS 4974, pp. 617-626, 2008. 